\title{
Evapotranspiração e estimativa da água consumida em perímetro irrigado do Semiárido brasileiro por sensoriamento remoto
}

\author{
Bernardo Barbosa da Silva(1), Alexandra Chaves Braga ${ }^{(2)}$, Célia Campos Braga ${ }^{(2)}$, Leidjane Maria Maciel de Oliveira(1), \\ Josiclêda Domiciano Galvíncio(1) e Suzana Maria Gico Lima Montenegro(1)
}

\begin{abstract}
(1)Universidade Federal de Pernambuco, Avenida Acadêmico Hélio Ramos, s/no, Cidade Universitária, CEP $50741-530$ Recife, PE. E-mail: bbdasilva.ufpe@gmail.com, leidjaneoliveira@hotmail.com, josicleda@hotmail.com, suzanam@ufpe.br (2)Universidade Federal de Campina Grande, Avenida Aprígio Veloso, no 882, Bairro Universitário, CEP 58429-120 Campina Grande, PB. E-mail: alexandra@dca.ufcg.edu.br, celia@dca.ufcg.edu.br
\end{abstract}

Resumo - O objetivo deste trabalho foi mapear a evapotranspiração real (ETr) e determinar o volume de água utilizado no projeto de irrigação de São Gonçalo, PB, no Semiárido brasileiro, com auxílio do sensoriamento remoto. Foram adquiridas imagens TM/Landsat 5 da área em estudo, em 2008, que foram utilizadas na obtenção dos mapas temáticos da ETr diária. Selecionaram-se dois pomares de coqueiro-anão para validação dos resultados da evapotranspiração obtida com o "surface energy balance algorithm for land" (ET $\left.T_{\text {Sebal }}\right)$ e com o método FAO-56 ( $\left.\mathrm{ET}_{\mathrm{FAO}}\right)$. Para determinação da $\mathrm{ET}_{\mathrm{FAO}}$, utilizou-se o produto entre o coeficiente de cultura, o coeficiente de ajuste e a evapotranspiração de referência. As diferenças obtidas, com uso das duas técnicas, resultaram em raiz do erro quadrado médio, erro relativo médio e erro absoluto médio iguais a $0,53 \mathrm{~mm}$, $9,46 \%$ e $0,43 \mathrm{~mm}$, respectivamente. Nas áreas irrigadas, a ETr representou $85 \%$ do saldo de radiação e, nas de sequeiro, apenas $12,5 \%$. O mapeamento da ETr delimitou claramente as áreas irrigadas das de sequeiro, bem como as diferenças existentes no interior do perímetro irrigado do projeto em investigação. O volume de água empregado no projeto de irrigação representa, somente no período de julho a dezembro, mais de $60 \%$ da capacidade do açude de São Gonçalo.

Termos para indexação: albedo, Landsat 5, saldo de radiação, temperatura da superfície.

\section{Evapotranspiration and assessment of water consumed in irrigated area of the Brazilian Semiarid Region by remote sensing}

\begin{abstract}
The objective of this work was to map the actual evapotranspiration (ETr) and to determine the volume of water used in the irrigation project of São Gonçalo, PB, in the Brazilian Semiarid Region, using remote sensing. $\mathrm{TM} /$ Landsat 5 images were acquired from the study area, in 2008, and were used to obtain thematic maps of daily ETr. Two dwarf coconut crops were selected for validation of the results of evapotranspiration obtained by the surface energy balance algorithm for land $\left(\mathrm{ET}_{\text {Sebal }}\right)$ and the FAO-56 method $\left(\mathrm{ET}_{\mathrm{FAO}}\right)$. In order to determine $\mathrm{ET}_{\mathrm{FAO}}$, the product between the culture coefficient, the adjustment coefficient, and the reference evapotranspiration was used. The differences obtained by the two techniques resulted in root mean square error, mean relative error, and mean absolute error equal to $0.53 \mathrm{~mm}, 9.46 \%$, and $0.43 \mathrm{~mm}$, respectively. In the irrigated areas, ETr represented about $85 \%$ of net radiation and, in rainfed plots, only $12.5 \%$. The mapping of ETr clearly delimitated the irrigated and nonirrigated plots, as well as the differences within the irrigated perimeter of the project under investigation. The volume of water applied in the irrigation project, only in the period from July to December, represents more than $60 \%$ of the capacity of the São Gonçalo reservoir.
\end{abstract}

Index terms: albedo, Landsat 5, net radiation, land surface temperature.

\section{Introdução}

Um dos maiores desafios da humanidade, no século 21, consiste na garantia do abastecimento de água potável e de alimentos aos 7 bilhões de habitantes do planeta, situação que deve ser agravada, pois projeções recentes indicam que, até 2050 , a população mundial pode superar 9 bilhões de pessoas (Bos et al.,
2005). Embora a produção mundial de alimentos ocorra majoritariamente no sistema de sequeiro, tem sido cada vez mais necessária a produção de alimentos mediante o uso da irrigação, que já representa $70 \%$ da água doce consumida no planeta (Fereres \& Soriano, 2007). A meta é produzir mais alimentos com uso de menor quantidade de água, tarefa que poderá ser bastante dificultada se forem confirmadas as projeções

Pesq. agropec. bras., Brasília, v.47, n.9, p.1218-1226, set. 2012 
de mudanças climáticas para as próximas décadas (Marengo et al., 2009).

Nos projetos de irrigação, no semiárido brasileiro, tem aumentado a competição pelo uso da água, que deve atender a outros setores da sociedade. A identificação do volume de água das irrigações possibilita avaliar a eficiência do seu uso e constitui elemento imprescindível à gestão sustentável dos recursos hídricos. Uma forma de contabilizar a água empregada na irrigação seria a instalação de hidrômetros em cada lote. Contudo, os elevados custos e a baixa operacionalidade dessa medida inviabilizam o seu uso. O sensoriamento remoto é uma opção de baixo custo e com grande cobertura que tem sido usada na obtenção da evapotranspiração real (ETr) das culturas (Kustas \& Norman, 1996; Bastiaanssen et al., 1998; Sobrino et al., 2005).

O uso da ETr, em vez do volume total de água utilizado nas irrigações, tem sido apontado como factível (Huffaker et al., 1998; Folhes et al., 2009), com base no argumento de que o excesso de água aplicada nas irrigações retornaria aos reservatórios, em razão da percolação da água. No entanto, esse procedimento é susceptível a críticas, já que poderia estimular ainda mais o desperdício de água e energia. Portanto, propõe-se o uso do sensoriamento remoto no cômputo da lâmina bruta de água, mensalmente, que depende da ETr das culturas e da eficiência do sistema de irrigação empregado.

Nas duas últimas décadas, foram desenvolvidas diferentes técnicas de sensoriamento remoto com vistas à determinação da ETr. Entre essas técnicas, destacamse o "surface energy balance algorithm for land" (SEBAL) (Bastiaanssen et al., 1998) e o "mapping evapotranspiration with internalized calibration" (METRIC) (Allen et al., 2007). O SEBAL tem se apresentado bastante preciso nas suas estimativas (Bastiaanssen et al., 1998; Folhes et al., 2009; Santos et al., 2010). O volume de água aplicado nas irrigações pode ser obtido ao se conjugar o volume de água resultante da evapotranspiração das culturas com o adicional da água perdida pelos sistemas de irrigação utilizados.

O objetivo deste trabalho foi mapear a evapotranspiração real (ETr) e determinar o volume de água utilizado no projeto de irrigação de São Gonçalo, PB, no semiárido brasileiro, com auxílio do sensoriamento remoto.

\section{Material e Métodos}

A área de estudo compreende o perímetro irrigado de São Gonçalo (PISG) (Figura 1). O recorte selecionado para a pesquisa apresenta 11,7 mil hectares, com destaque para dois pomares de coqueiro-anão, área de sequeiro, o Rio Piranhas e o açude de São Gonçalo. Nas áreas dos dois pomares de coqueiro (com idade superior a cinco anos), utilizou-se espaçamento entre plantas de 7,5 m, em configuração triangular. A irrigação foi feita predominantemente por microaspersão. A área denominada sequeiro compreende a área de pastagem em regime de sequeiro.

O clima da região é classificado como semiárido, tipologia DdA', segundo Thornthwaite (Varejão-Silva et al., 1984), com estação chuvosa concentrada no período de fevereiro a maio. As médias anuais de temperatura do ar, precipitação e evapotranspiração potencial (ETp) são, respectivamente, $25,3^{\circ} \mathrm{C}$, 995,5 mm e $1.480 \mathrm{~mm}$. Predominam, no PISG, os solos Latossolos e Cambissolos.

Para a obtenção do balanço de energia e da evapotranspiração com o SEBAL, foram obtidas imagens do mapeador temático (TM) do satélite Landsat 5, órbita 216 e ponto 65, no Instituto de Pesquisas Espaciais. Essas imagens são compostas de sete bandas espectrais com resolução espacial de $30 \times 30 \mathrm{~m}$, exceto a banda 6 (banda termal), com resolução de 120x120 m. A passagem do Landsat 5, na área de estudo, ocorreu aproximadamente às $9 \mathrm{~h} 30 \mathrm{~min}$ (tempo local) nos dias avaliados (29 de agosto, 14 de setembro, 1 e 17 de novembro, e 19 de dezembro de 2008). Os dados utilizados no cômputo dos diferentes componentes do balanço de radiação são apresentados na Tabela 1.

No SEBAL, a evapotranspiração real diária (ETc) é obtida a partir do fluxo de calor latente (LE), que é calculado como resíduo do balanço de energia à superfície:

$\mathrm{LE}=\mathrm{R}_{\mathrm{n}}-\mathrm{G}-\mathrm{H}$

em que $R_{n}\left(W m^{-2}\right)$ é o saldo de radiação; $G\left(W m^{-2}\right)$ é o fluxo de calor no solo; e $\mathrm{H}\left(\mathrm{W} \mathrm{m}^{-2}\right)$ é o de fluxo de calor sensível. Determinou-se o $\mathrm{R}_{\mathrm{n}}$ em cada pixel da área de estudo, conforme a equação (Allen et al., 2007):

$\mathrm{R}_{\mathrm{n}}=\mathrm{R}_{\mathrm{s} \text {,inst }}(1-\alpha)+\varepsilon \mathrm{R}_{\mathrm{ol}, \text { atm }}-\mathrm{R}_{\mathrm{ol}, \mathrm{emi}}$

em que $\mathrm{R}_{\mathrm{s}, \text { inst }}\left(\mathrm{W} \mathrm{m}^{-2}\right)$ é a radiação solar global instantânea; $\alpha$ (adimensional) é o albedo da superfície; 
$\mathrm{R}_{\mathrm{ol}, \mathrm{atm}}\left(\mathrm{W} \mathrm{m}^{-2}\right)$ é a radiação de onda longa emitida pela atmosfera na direção da superfície; $\mathrm{R}_{\mathrm{ol}, \mathrm{emi}}\left(\mathrm{W} \mathrm{m}^{-2}\right)$ é a radiação de onda longa emitida pela superfície; e $\varepsilon$ (adimensional) é a emissividade da superfície. Todos os detalhes relativos ao cômputo de cada uma das componentes do balanço de radiação se encontram em Silva et al. (2005). Já o fluxo de calor no solo (G), foi obtido por meio de modelo proposto por Bastiaanssen (2000):

$\mathrm{G}=\left[\mathrm{T}_{\text {sup }}(0,0038+0,0074 \alpha)\left(1-0,98 \mathrm{IVDN}^{4}\right)\right] \mathrm{R}_{\mathrm{n}}$

em que: $\mathrm{T}_{\text {sup }}\left({ }^{\circ} \mathrm{C}\right)$ é a temperatura da superfície; $\alpha$ (adimensional) é o albedo; IVDN (adimensional) é o índice de vegetação da diferença normalizada; e $\mathrm{R}_{\mathrm{n}}\left(\mathrm{W} \mathrm{m}^{-2}\right)$ é o saldo de radiação em cada pixel da imagem. Para efeito de cálculo, assumiu-se $G=0,3 R_{n}$ em corpos de água (IVDN<0) (Silva et al., 2005;
Bezerra et al., 2008). Os valores do fluxo de calor sensível $(\mathrm{H})$ foram estimados com base na velocidade do vento e na diferença da temperatura do ar (dT) entre dois níveis próximos da superfície $\left(Z_{1}=0,1 \mathrm{~m}\right.$ e $\mathrm{Z}_{2}=2 \mathrm{~m}$ ), de acordo com o SEBAL (Bastiaanssen et al., 1998; Allen et al., 2007),

$H=\rho \times c_{p}\left(d T / r_{a h}\right)$

em que: $\rho\left(\mathrm{kg} \mathrm{m}^{-3}\right)$ é a densidade do ar atmosférico; $\mathrm{c}_{\mathrm{p}}\left(\mathrm{kJ} \mathrm{kg}^{-1} \mathrm{~K}^{-1}\right)$ é o calor específico do ar à pressão constante; e $r_{a h}\left(\mathrm{~s} \mathrm{~m}^{-1}\right)$ é a resistência aerodinâmica ao transporte de calor sensível. Uma importante hipótese do SEBAL consiste em considerar linear a diferença entre a temperatura do ar (dT) e a temperatura da superfície, ou seja: $d \mathrm{~T}=\mathrm{a}+\mathrm{b} \mathrm{T}_{\text {sup}}$, em que as constantes de calibração a e b requerem, para a sua determinação, a identificação de dois pixels de referência (pixels âncoras) que representam condições extremas de

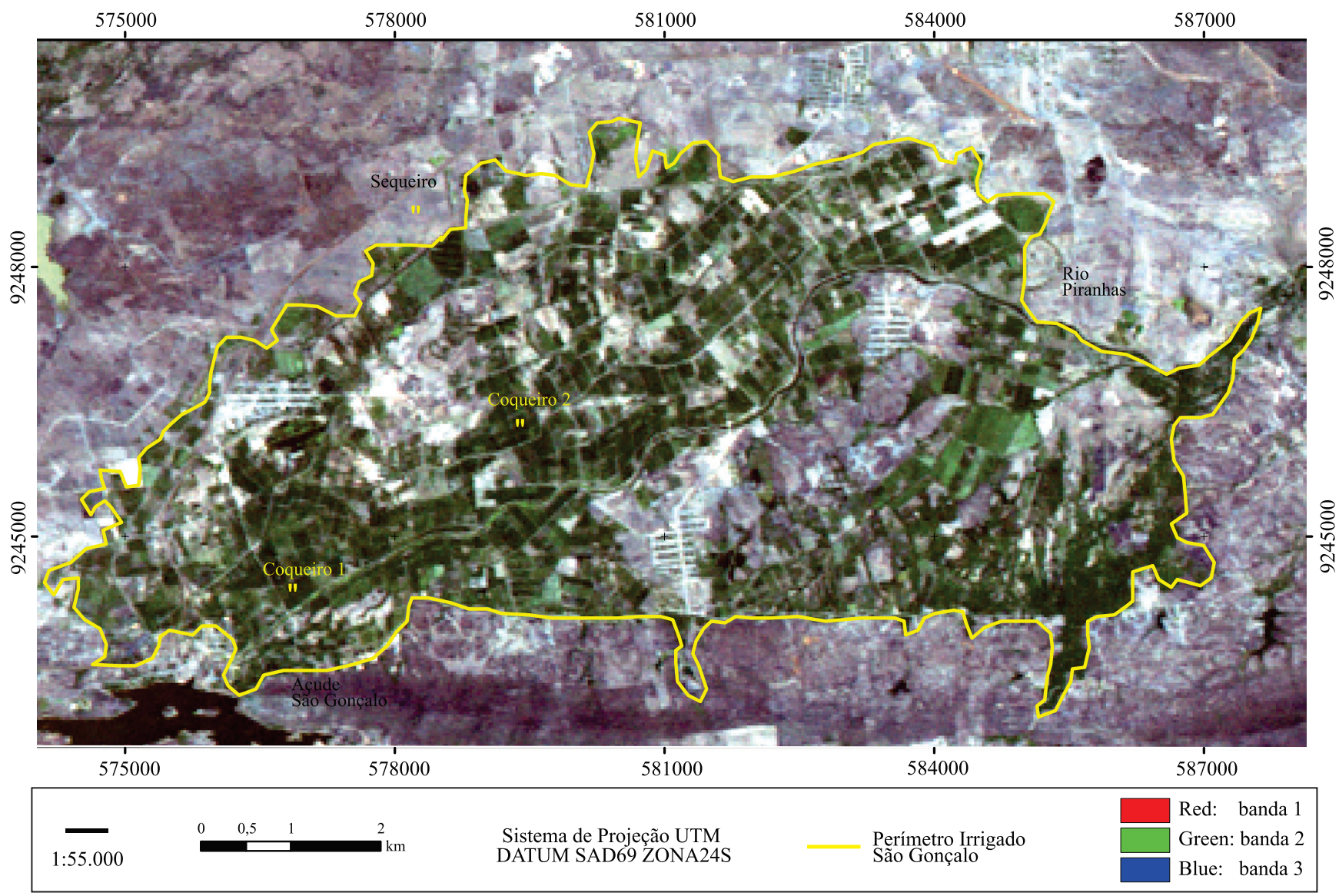

Figura 1. Recorte da área de estudo com destaque para áreas de coqueiro-anão selecionadas para validação, área de sequeiro, perímetro irrigado de São Gonçalo, Rio Piranhas e açude de São Gonçalo, em combinação RGB123 de imagem TM/ Landsat 5 , de $1 / 11 / 2008$. 
temperatura e umidade, denominados pixels quente e frio. O pixel frio localizou-se em um ponto do açude de São Gonçalo, onde se assumiu $\mathrm{H}=0$ (Bastiaanssen et al., 1998). Já o pixel quente, foi escolhido em área de solo com grande exposição, onde se assumiu $\mathrm{LE}=0$, ou seja, $H=R_{n}$ - $G$, o que tornou possível obter os valores das constantes de calibração (Bastiaanssen et al., 1998; Bezerra et al., 2008). Esses valores iniciais de $\mathrm{H}$ servem apenas como parâmetros de entrada de processo iterativo, em que é identificada a condição de estabilidade de cada pixel, mediante teoria da similaridade de Monin-Obukhov e valor do parâmetro $\mathrm{L}(\mathrm{m})$,

$\mathrm{L}=-\rho \times \mathrm{c}_{\mathrm{p}} \times \mathrm{u}_{*}^{3} \times \mathrm{T}_{\text {sup }} / \mathrm{k} \times \mathrm{g} \times \mathrm{H}$

em que: $u_{*}\left(\mathrm{~m} \mathrm{~s}^{-1}\right)$ é a velocidade de fricção; $T_{\text {sup }}(\mathrm{K})$ é a temperatura da superfície; $\mathrm{k}=0,41$ (adimensional) é a constante de von Karman; $\mathrm{g}\left(\mathrm{m} \mathrm{s}^{-2}\right)$ é o módulo do campo gravitacional terrestre; e $\mathrm{H}\left(\mathrm{W} \mathrm{m}^{-2}\right)$ é o fluxo de calor sensível, obtido, inicialmente, ao se considerar a atmosfera em condição de estabilidade neutra. Dependendo da condição de estabilidade da atmosfera, são aplicadas correções aos novos valores de $\mathrm{u}_{*}$ e $\mathrm{r}_{\mathrm{ah}}$, com os quais são obtidos novos valores das constantes de calibração de dT. O processo continua até que se alcance a estabilidade do processo iterativo. Em seguida, são obtidos um mapa temático de $\mathrm{H}$ e a densidade do fluxo de calor latente $\left(\mathrm{W} \mathrm{m}^{-2}\right)$, em cada pixel da área de estudo (Silva \& Bezerra, 2006; Bezerra et al., 2008).

Os valores da evapotranspiração real diária (ETr) foram obtidos a partir de extrapolação do valor instantâneo do LE (equação 1), por meio da expressão (Bezerra et al., 2008),

Tabela 1. Datas das imagens do mapeador temático, ângulo zenital do sol ( $Z$, grau), quadrado da razão entre a distância Terra-Sol e seu valor médio (dr), cosseno do ângulo zenital solar $(\cos \mathrm{Z})$, temperatura do ar $\left(\mathrm{Ta},{ }^{\circ} \mathrm{C}\right)$, umidade relativa (UR, \%), pressão atmosférica $\left(\mathrm{p}_{\mathrm{o}}, \mathrm{KPa}\right)$, radiação solar instantânea $\left(\mathrm{R}_{\mathrm{s}, \text { inst }}, \mathrm{W} \mathrm{m}^{-2}\right)$ e transmitância atmosférica $\left(\tau_{\mathrm{oc} \text {,ins, }}\right.$, adimensional), no momento da passagem do satélite, na área de estudo, em 2008.

\begin{tabular}{lcccccccc}
\hline Data & $\begin{array}{c}\mathrm{Z} \\
(\mathrm{grau})\end{array}$ & $\mathrm{d}_{\mathrm{r}}$ & $\cos \mathrm{Z}$ & $\begin{array}{c}\mathrm{Ta} \\
\left({ }^{\circ} \mathrm{C}\right)\end{array}$ & $\begin{array}{c}\mathrm{UR} \\
(\%)\end{array}$ & $\begin{array}{c}\mathrm{p}_{\mathrm{o}} \\
(\mathrm{kPa})\end{array}$ & $\begin{array}{c}\mathrm{R}_{\text {s,inst }} \\
(\mathrm{W} \mathrm{m})\end{array}$ & $\tau_{\text {oc,ins }}$ \\
\hline 29 de agosto & 35,30 & 0,9828 & 0,8161 & 29,1 & 46,2 & 98,1 & 808 & 0,737 \\
14 de setembro & 31,84 & 0,9912 & 0,8495 & 29,2 & 47,1 & 98,9 & 851 & 0,740 \\
1 de novembro & 27,94 & 1,0174 & 0,8834 & 29,5 & 45,0 & 98,7 & 914 & 0,744 \\
17 de novembro & 29,48 & 1,0244 & 0,8705 & 28,6 & 46,3 & 98,3 & 909 & 0,745 \\
19 de dezembro & 33,53 & 1,0324 & 0,8336 & 29,6 & 47,5 & 93,4 & 867 & 0,737 \\
\hline
\end{tabular}

$\mathrm{ETr}=0,035(\mathrm{LE} / \mathrm{Rn}-\mathrm{G})\left[(1-\alpha) \mathrm{R}_{\mathrm{s}, 24 \mathrm{~h}}-123 \tau_{\mathrm{oc}, 24}\right]$

em que: $\mathrm{R}_{\mathrm{s}, 24 \mathrm{~h}}$ é a radiação solar global diária $\left(\mathrm{W} \mathrm{m}^{-2}\right)$; $\tau_{\mathrm{oc}, 24 \mathrm{~h}}$ (adimensional) é a transmissividade atmosférica média diária, obtida com base na radiação solar global diária observada na estação meteorológica e na radiação solar diária do topo da atmosfera terrestre nos dias avaliados; e 0,035 é o fator de conversão de $\mathrm{W} \mathrm{m}^{-2}$ para $\mathrm{mm}$ por dia.

Para a análise da precisão das estimativas da $\mathrm{ET}_{\text {Sebal, }}$, foram utilizados o erro absoluto médio (EMA), o erro relativo médio (ERM) e a raiz do erro quadrado médio (REQM),

$$
\begin{aligned}
& E M A=\frac{1}{N} \sum_{i=1}^{N}\left|E_{\text {sebal }}-E_{\mathrm{FAO}}\right| \\
& \mathrm{EMA}=\frac{100}{\mathrm{~N}} \sum_{\mathrm{i}=1}^{\mathrm{N}}\left|\frac{\mathrm{ET}_{\text {sebal }}-\mathrm{ET}_{\mathrm{FAO}}}{\mathrm{ET}_{\mathrm{FAO}}}\right| \\
& \mathrm{REQM}=\left(\frac{\sum_{\mathrm{i}=1}^{\mathrm{N}}\left(\mathrm{ET}_{\text {sebal }}-\mathrm{ET}_{\mathrm{FAO}}\right)^{2}}{\mathrm{~N}}\right)
\end{aligned}
$$

em que: $\mathrm{ET}_{\text {Sebal }}$ e $\mathrm{ET}_{\mathrm{FAO}}$ correspondem, respectivamente, aos valores da evapotranspiração segundo o SEBAL e o método da $\mathrm{FAO}$, e $\mathrm{N}$ é o número de pares dessas variáveis.

Os dados diários de temperaturas máxima e mínima, umidades relativas do ar máxima e mínima, pressão atmosférica média diária, velocidade do vento e radiação solar global diária, obtidos na estação automática do Instituto Nacional de Meteorologia, localizada em São Gonçalo, PB, foram utilizados para obtenção da evapotranspiração de referência diária segundo o métodoFAO-56 de Penman-Monteith (Allen et al., 1998), para todo o ano de 2008. Com base no coeficiente de cultura $(\mathrm{Kc})$ do coqueiro-anão (Miranda et al., 2007; Sousa et al., 2011), obteve-se a $\mathrm{ET}_{\mathrm{FAO}}$, pelo produto ETo x Kc x Ka (Allen et al., 1998), em que Ka é um coeficiente de ajuste dependente do fator de cobertura do solo. Esse método de determinação da evapotranspiração real de cultivos irrigados é o mais empregado nas práticas de irrigação e foi utilizado no procedimento de validação do SEBAL, para os dias com imagens disponíveis, em 2008.

Para a determinação do volume total de água aplicada mensalmente nas parcelas irrigadas do PISG, fez-se um recorte contornando o referido perímetro (Figura 1) e admitiu-se que as parcelas irrigadas compreendiam todos os pixels com IVDN $>0,4$, o que resultou em área

Pesq. agropec. bras., Brasília, v.47, n.9, p.1218-1226, set. 2012 
total irrigada média de 3.559 ha. Em seguida, gerou-se arquivo em ASCII e obteve-se, em planilha eletrônica, a relação de todos os pixels do recorte correspondente ao PISG, para determinação da evapotranspiração real diária acumulada (ETr_acum_24h) de todos os 39.500 pixels de cada imagem selecionada para a pesquisa. Com base na hipótese de que a razão entre a ETr acumulada diária e a ETo acumulada diária se manteria constante ao longo do mês, obteve-se a evapotranspiração real acumulada mensal (ETr_Acum mensal) pelo produto dessa razão pela ETo mensal.

\section{Resultados e Discussão}

Os dados complementares às imagens do TM/ Landsat 5, utilizados nos balanços de radiação e energia com o SEBAL, estão descritos nas Tabelas 1 e 2. Observou-se que, nos dias com baixa presença de nebulosidade, a temperatura do ar manteve-se entre 21,8 e $36,1^{\circ} \mathrm{C}$, enquanto a umidade relativa do ar variou de 27 a 92\%. A velocidade média diária do vento apresentou-se bastante estável, entre 1,27 e 1,59 $\mathrm{m} \mathrm{s}^{-1}$, semelhantemente à pressão atmosférica, que também foi pouco variável, com valor médio de 98,33 KPa. Já a radiação solar global diária, manteve-se entre 25,27 e $28,85 \mathrm{MJ} \mathrm{m}^{-2}$, e a evapotranspiração de referência (ETo) esteve entre 5,4 e 6,1 mm, em 14 de setembro e 19 de dezembro, respectivamente.

Foram observadas diferenças significativas entre os padrões das áreas irrigadas e os das áreas adjacentes nos mapas temáticos dos fluxos de calor no solo $(\mathrm{G})$, calor sensível $(\mathrm{H})$ e calor latente (LE) (Figura 2). Os valores de $G$ foram, em geral, inferiores a $150 \mathrm{~W} \mathrm{~m}^{-2}$, exceto nos corpos de água onde chegaram a 0,3 do saldo de radiação $\left(R_{n}\right)$. Nas áreas com elevados valores de $\mathrm{H}$, houve baixos valores de LE.
As áreas em verde mais intenso (Figura 3) compreendem os maiores valores de ETr, com destaque para o açude de São Gonçalo e as grandes extensões do PISG. Os padrões evapotranspirativos das áreas irrigadas, sejam os do PISG ou os de áreas próximas ao leito do Rio do Peixe, localizadas no canto superior esquerdo, mudaram pouco ao longo dos dias avaliados. Nas áreas irrigadas, prevaleceram valores maiores que $3 \mathrm{~mm}$ e, nas áreas de sequeiro e ocupadas com habitações humanas, foram observados valores entre 0 e $2 \mathrm{~mm}$. Em 19 de dezembro, detectou-se expansão das áreas com maiores valores da ETr, principalmente no PISG, mas, também, nas áreas de sequeiro que apresentaram aumento considerável na ETr. Pode-se relacionar esse aumento, nas áreas irrigadas, ao aumento no saldo de radiação. No caso das áreas de sequeiro, este aumento estaria associado à ocorrência das primeiras chuvas da estação chuvosa, que se inicia em dezembro. Em 1 de novembro e 19 de dezembro, a intensidade do verde foi visualmente maior, o que indica maior número de pixels com valores da ETr na faixa de 5,3 a $6 \mathrm{~mm}$ por dia (Tabelas 3 e 4).

Verificou-se que, na área de coqueiro 1, o albedo variou de apenas 0,154 (29 de agosto) a 0,177 (17 de novembro), enquanto, na área de coqueiro 2 , este manteve-se entre 0,142 (14 de setembro) e 0,175 (17 de novembro) (Tabela 3). Portanto, na área de sequeiro, não submetida à irrigação, o albedo apresentou valores bem mais elevados: de 0,196 (14 de setembro) a 0,311 (29 de agosto). Silva et al. (2005) também constataram diferenças significativas no albedo de áreas irrigadas e não irrigadas, bem como a influência da correção atmosférica no cômputo do albedo de áreas heterogêneas no semiárido brasileiro.

O saldo de radiação é de grande importância em estudos relacionados às trocas de energia e massa

Tabela 2. Datas das imagens do mapeador temático, temperaturas do ar máxima (Tmax, $\left.{ }^{\circ} \mathrm{C}\right)$ e mínima $\left(\mathrm{Tmin},{ }^{\circ} \mathrm{C}\right)$ diárias, umidades relativas máxima (URmax, \%) e mínima (URmin, \%) diárias, velocidade média diária do vento (vv, $\mathrm{m} \mathrm{s}^{-1}$ ) a $2 \mathrm{~m}$, pressão atmosférica média diária $\left(\mathrm{p}_{\mathrm{o}}, \mathrm{kPa}\right)$, radiação solar global diária $\left(\mathrm{R}_{\mathrm{s}, 24 \mathrm{~h}}, \mathrm{MJ} \mathrm{m}^{-2}\right)$, evapotranspiração de referência FAO-56 (ETo, mm) e radiação solar diária no topo da atmosfera $\left(\mathrm{R}_{\mathrm{s}, \text { toa }}, \mathrm{MJ} \mathrm{m}^{-2}\right)$, referentes aos dias selecionados para o estudo, em São Gonçalo, PB, em 2008.

\begin{tabular}{|c|c|c|c|c|c|c|c|c|c|}
\hline Data & Tmax & Tmin & URmax & URmin & vV & $\mathrm{p}_{\mathrm{o}}$ & $\mathrm{R}_{\mathrm{s}, 24 \mathrm{~h}}$ & ETo & $\mathrm{R}_{\mathrm{s}, \text { toa }}$ \\
\hline 29 de agosto & 32,4 & 22,6 & 73 & 57 & 1,57 & 98,16 & 25,48 & 5,5 & 35,29 \\
\hline 14 de setembro & 32,2 & 22,0 & 77 & 65 & 1,57 & 98,63 & 25,27 & 5,4 & 36,71 \\
\hline 1 de novembro & 32,5 & 24,0 & 87 & 66 & 1,59 & 98,52 & 27,40 & 5,9 & 38,51 \\
\hline 17 de novembro & 33,1 & 25,4 & 92 & 76 & 1,49 & 98,26 & 27,48 & 5,8 & 38,43 \\
\hline 19 de dezembro & 36,1 & 21,8 & 84 & 27 & 1,27 & 98,10 & 28,85 & 6,1 & 38,28 \\
\hline
\end{tabular}

Pesq. agropec. bras., Brasília, v.47, n.9, p.1218-1226, set. 2012 

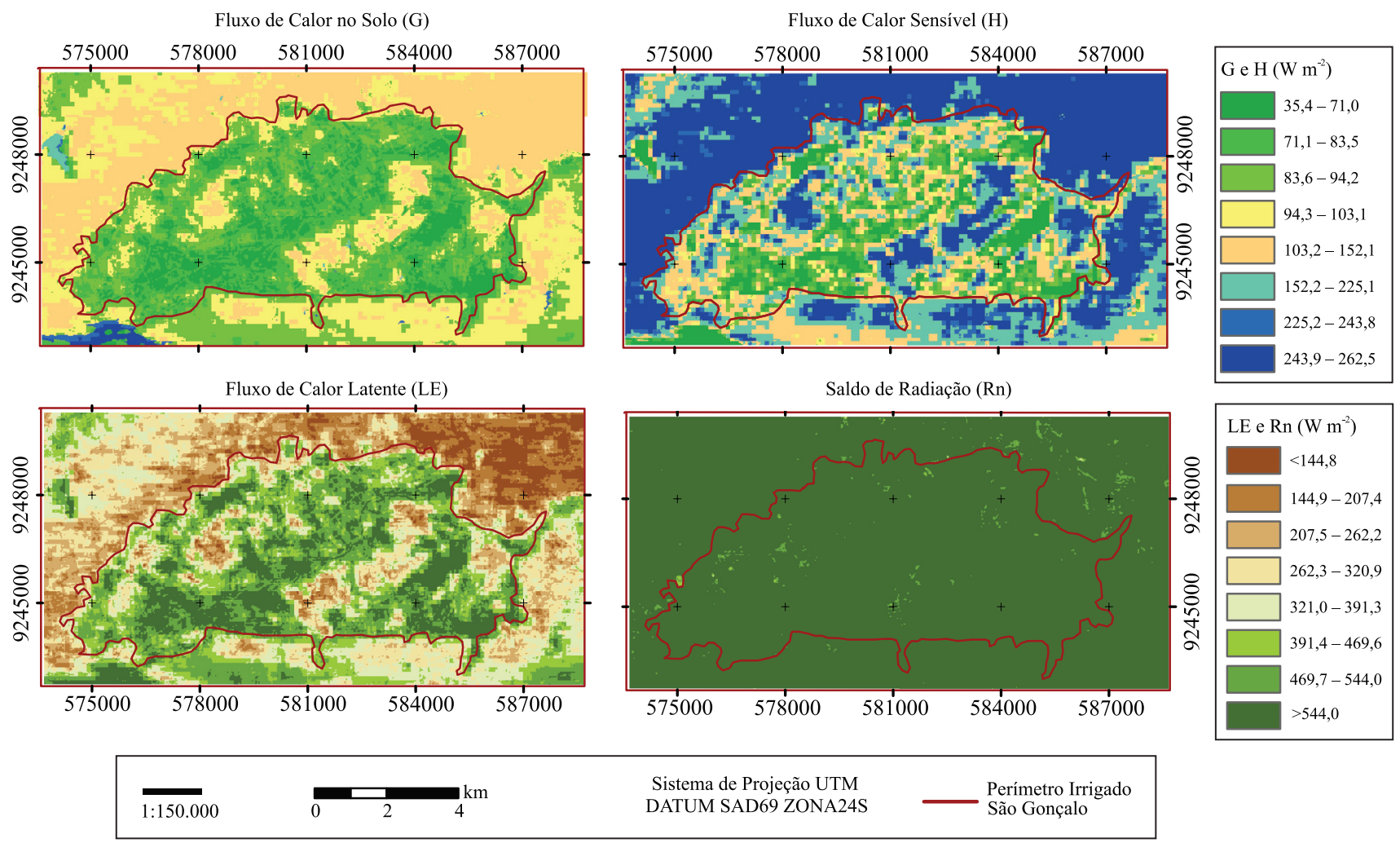

Figura 2. Mapas temáticos dos fluxos de calor no solo $(\mathrm{G})$, calor sensível (H), calor latente (LE) e saldo de radiação (Rn), para 1/11/2008, obtidos por meio do SEBAL, em São Gonçalo, PB.
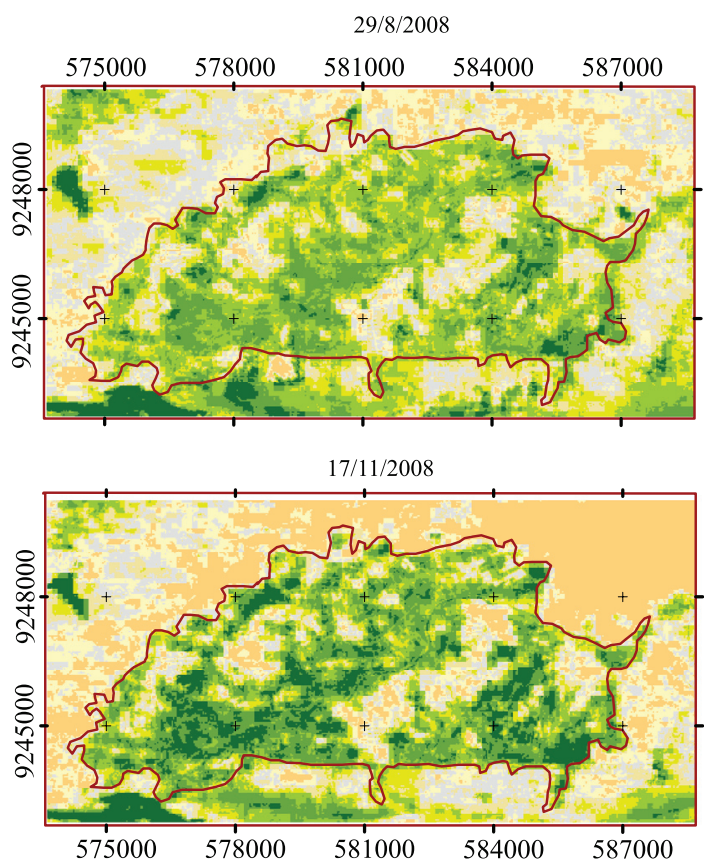
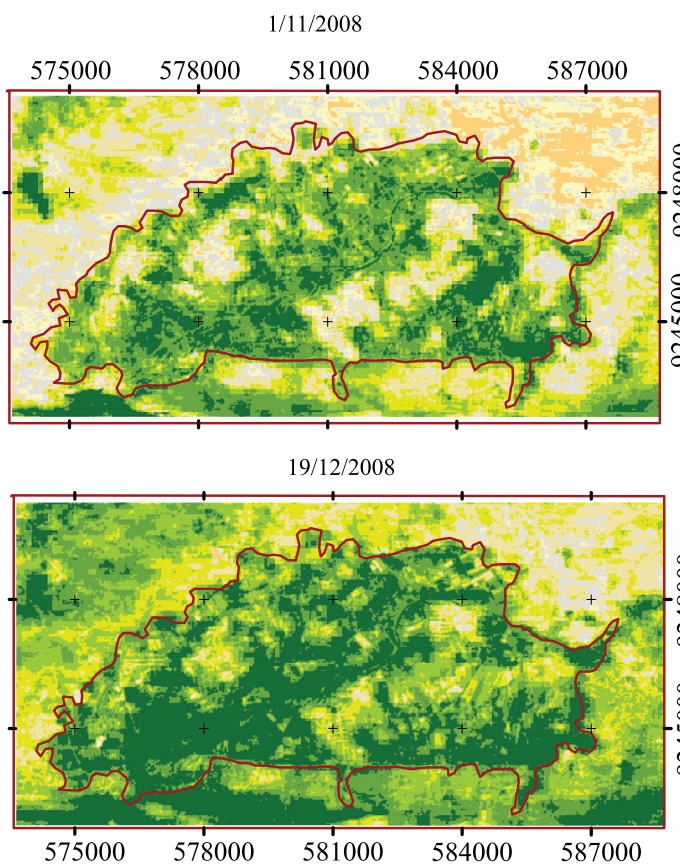

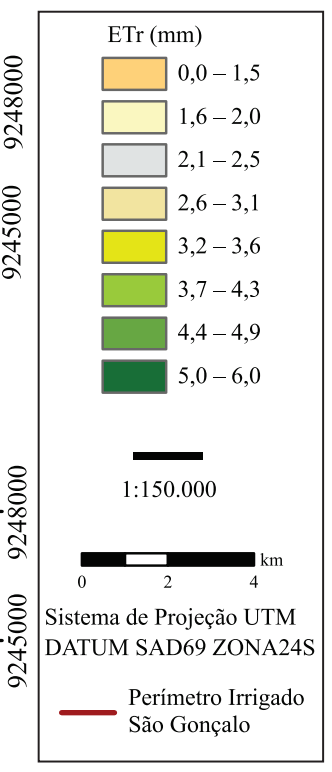

Figura 3. Mapas temáticos da evapotranspiração real diária (ETr) (mm) para 29 de agosto, 1 e 17 de novembro e 19 de dezembro, de 2008, em São Gonçalo, PB. 
entre a superfície terrestre e a atmosfera, e é essencial em várias aplicações, inclusive no monitoramento e previsão de tempo, na modelagem hidrológica e na meteorologia agrícola (Silva et al., 2005; Bezerra et al., 2008). Nos pomares de coqueiro irrigados no PISG, o $\mathrm{R}_{\mathrm{n} 24 \mathrm{~h}}$ foi superior ao observado nas áreas de sequeiro, em particular naquelas com baixa cobertura vegetal. Nas áreas de coqueiro 1 e 2, o $\mathrm{R}_{\mathrm{n} 24 \mathrm{~h}}$ médio foi de 153 e $154,7 \mathrm{~W} \mathrm{~m}^{-2}$, respectivamente, enquanto, na área de sequeiro, foi de $124,7 \mathrm{~W} \mathrm{~m}^{-2}$ (Tabela 3). As maiores diferenças entre o pomar de coqueiros e a área de sequeiro foram observadas em 29 de agosto, quando o $\mathrm{R}_{\mathrm{n} 24 \mathrm{~h}}$ alcançou $146,8 \mathrm{~W} \mathrm{~m}^{-2}$, na área de coqueiro 2 , e apenas $100 \mathrm{~W} \mathrm{~m}^{-2}$ na de sequeiro.

Silva et al. (2005) relataram valores próximos para o lago de Sobradinho na Bahia. Já Bastiaanssen et al. (1998), obtiveram $R_{n}$ de $650 \mathrm{~W} \mathrm{~m}^{-2}$, em oásis, e de $400 \mathrm{~W} \mathrm{~m}^{-2}$, nas áreas desérticas, na Bacia do Rio Heihe, na China. Em outro estudo na Bacia de Gediz, na Turquia, Bastiaanssen (2000) observou valores de $\mathrm{R}_{\mathrm{n}}$ entre 300 e $600 \mathrm{~W} \mathrm{~m}^{-2}$. No presente trabalho, nas áreas irrigadas com elevados índices de vegetação, particularmente com IVDN $>0,6$, cerca de $50 \%$ da radiação solar global diária $\left(\mathrm{R}_{\mathrm{s} 24 \mathrm{~h}}\right)$ foi empregada no processo evapotranspirativo, enquanto, nas áreas de sequeiro com pequena cobertura vegetal, apenas $11,2 \%$ da $R_{\mathrm{s} 24 \mathrm{~h}}$ foi utilizada no processo evapotranspirativo. Observou-se que, em média, $84,7 \%$ do $\mathrm{R}_{\mathrm{n}}$ foi empregado na evapotranspiração dos coqueiros irrigados no PISG, o que está em concordância com diversos estudos que mostraram, por meio do balanço de energia, que a razão entre $L E$ e $R_{n}$ situa-se entre 78 e $85 \%$ (Silva et al., 2007; Borges et al., 2008).

Para a validação da evapotranspiração obtida com o SEBAL no PISG, foram considerados dois pomares de coqueiro irrigados (Tabela 3) e a evapotranspiração derivada do método Kc x Ka x ETo (Allen et al., 1998), denominado aqui de $\mathrm{ET}_{\mathrm{FAO}}$. Embora o coqueiro irrigado após o quinto ano de idade, no semiárido brasileiro, apresente coeficiente de cultura $(\mathrm{Kc})=1,0$ (Miranda et al., 2007; Sousa et al., 2011), também é necessário considerar o coeficiente de ajuste (Ka) (Allen et al., 1998) que depende da fração de cobertura do solo. Esse valor, no quinto ano de idade do coqueiro-anão, foi igual a 0,8 (Miranda et al., 2007), o que resultou em REQM, ERM e EAM iguais a $0,53 \mathrm{~mm}, 9,46 \%$ e $0,43 \mathrm{~mm}$, respectivamente (Tabela 3). No entanto, ao se simular a $\mathrm{ET}_{\mathrm{FAO}}$ com $\mathrm{Ka}=0,9$, esses erros foram reduzidos a $0,35 \mathrm{~mm}, 5,61 \%$ e $0,28 \mathrm{~mm}$, respectivamente. Santos et al. (2010) obtiveram EAM igual a $0,3 \mathrm{~mm}$ por dia ao

Tabela 3. Datas das imagens do mapeador temático, evapotranspiração de referência $\left(\mathrm{ET}_{\mathrm{o}}, \mathrm{mm}\right)$, albedo ( $\alpha$, adimensional), saldo de radiação diário $\left(\mathrm{R}_{\mathrm{n} 24 \mathrm{~h}}, \mathrm{~W} \mathrm{\textrm {m } ^ { - 2 } )}\right.$ e evapotranspiração real diária SEBAL $\left(\mathrm{ET}_{\text {Sebal, }}, \mathrm{mm}\right)$ referentes aos dias avaliados em São Gonçalo, PB, em 2008.

\begin{tabular}{|c|c|c|c|c|c|c|c|c|c|c|c|c|}
\hline \multirow[t]{2}{*}{ Data } & \multirow[t]{2}{*}{ ETo } & \multicolumn{4}{|c|}{ Área de coqueiro 1} & \multicolumn{4}{|c|}{ Área de coqueiro 2} & \multicolumn{3}{|c|}{ Área de sequeiro } \\
\hline & & $\alpha$ & $\mathrm{R}_{\mathrm{n} 24 \mathrm{~h}}$ & $\mathrm{ET}_{\text {Sebal }}$ & $\mathrm{ET}_{\mathrm{FAO}}$ & $\alpha$ & $\mathrm{R}_{\mathrm{n} 24 \mathrm{~h}}$ & $\mathrm{ET}_{\text {Sebal }}$ & $\mathrm{ET}_{\mathrm{FAO}}$ & $\alpha$ & $\mathrm{R}_{\mathrm{n} 24 \mathrm{~h}}$ & $\mathrm{ET}_{\text {Sebal }}$ \\
\hline 29 de agosto & 5,5 & 0,154 & 146,2 & 4,4 & 4,4 & 0,152 & 146,8 & 5,1 & 4,4 & 0,311 & 100,0 & 0,6 \\
\hline 14 de setembro & 5,4 & 0,159 & 147,5 & 4,6 & 4,3 & 0,142 & 152,5 & 5,6 & 4,3 & 0,196 & 142,7 & 1,0 \\
\hline 1 de novembro & 5,9 & 0,166 & 162,7 & 5,1 & 4,7 & 0,168 & 162,1 & 5,1 & 4,7 & 0,270 & 129,8 & 1,7 \\
\hline 17 de novembro & 5,8 & 0,177 & 159,5 & 5,0 & 4,7 & 0,175 & 160,1 & 5,0 & 4,7 & 0,287 & 124,5 & 1,3 \\
\hline 19 de dezembro & 6,1 & 0,170 & 149,3 & 5,1 & 4,9 & 0,162 & 152,0 & 5,2 & 4,9 & 0,254 & 126,6 & 1,4 \\
\hline
\end{tabular}

Tabela 4. Valores de evapotranspiração de referência FAO-56 mensal (ETo) e real acumulada mensal (ETr Acum mensal), razão RETr/ETo e volume total evapotranspirado mensalmente, no período de maior demanda de água, na área total irrigada (ATI) do perímetro irrigado de São Gonçalo, em 2008.

\begin{tabular}{lcccc}
\hline Mês & ATI (ha) & $\begin{array}{c}\text { ETo } \\
(\mathrm{mm} \text { por mês })\end{array}$ & $\begin{array}{c}\text { ETr Acum mensal } \\
(\mathrm{mm} \text { por mês })\end{array}$ & $\begin{array}{c}\text { RETr/ETo } \\
\text { Volume evapotranspirado } \\
\left(\mathrm{m}^{3} \text { por mês }\right)\end{array}$ \\
\hline Julho & 3.552 & 126,4 & $151.500,1$ & $3.133 .777,2$ \\
Agosto & 3.552 & 154,3 & $151.500,3$ & 0,693 \\
Setembro & 3.551 & 161,7 & $147.341,2$ & 0,693 \\
Outubro & 3.552 & 173,0 & $159.396,0$ & 0,693 \\
Novembro & 3.573 & 174,9 & $171.450,8$ & 0,719 \\
Dezembro & 3.572 & 177,7 & $197.137,4$ & 0,744 \\
\hline
\end{tabular}


comparar a $\mathrm{ET}_{\text {Sebal }}$ com medições feitas com o balanço de energia baseado na razão de Bowen (BERB). Portanto, as condições de cultivo dos dois pomares irrigados, selecionados para a validação, indicam que o Ka seria igual a 0,9. Contudo, este resultado deve ser confirmado por meio de campanha experimental específica, na qual sejam adotadas outras técnicas de obtenção da ETr em pomares do PISG. Além disso, ao se considerar $K a=0,9$, a razão entre $E T r$ versus $R_{n, 24 h}$ aumenta para mais de $90 \%$.

Nos pomares de coqueiro irrigados, a $\mathrm{ET}_{\text {Sebal }}$ variou de 4,4 a 5,6 mm (Tabela 3). Na área de sequeiro, a $\mathrm{ET}_{\text {Sebal }}$ apresentou valor mínimo de $0,6 \mathrm{~mm}$ e máximo de $1,7 \mathrm{~mm}$. Bezerra et al. (2008) validaram o SEBAL com a ETr derivada do BERB, em cultivos de algodão e mamona no Cariri cearense, e obtiveram ERM e REQM de $6,48 \%$ e $0,35 \mathrm{~mm}$ por dia, respectivamente. Outros autores relataram diferenças entre a $\mathrm{ET}_{\text {Sebal }}$ e as medições de campo, em geral, inferiores a $10 \%$ (Timmermans et al., 2007; Zwart \& Bastiaanssen, 2007). Esses resultados são indicativos do bom desempenho da técnica em condições edafoclimáticas diversas.

O PISG reúne, em média, 3.559 ha irrigados, segundo os critérios estabelecidos no presente trabalho. Observou-se que o volume de água evapotranspirado mensalmente situou-se entre $3.133 .772 \mathrm{~m}^{3}$ (em julho) e $5.167 .124 \mathrm{~m}^{3}$ (em dezembro), com total, no semestre de maior demanda por irrigação, de $25.123 .130 \mathrm{~m}^{3}$ (Tabela 4).

A ETo mensal, no semestre de maior demanda por irrigação (julho a dezembro), variou de $126,4 \mathrm{~mm}$, em julho, a 177,7 mm em dezembro. Nos dias com imagens $\mathrm{TM} /$ Landsat 5, constatou-se que a ETr acumulada em todos os pixels com IVDN $>0,4$ situou-se entre $147.341 \mathrm{~mm}$ (em setembro) e $197.137 \mathrm{~mm}$ (em dezembro). Em julho e outubro, não foi possível a utilização de imagens, em razão da grande presença de nuvens. Nesse sentido, considerou-se, para julho, o valor da RETr_ETo de agosto e a sua média entre setembro e novembro como representativo da razão em outubro. Esta razão flutuou de 0,693 (agosto) a 0,814 (dezembro) e resultou em volume de água evapotranspirado mensalmente igual a $3.133 .772 \mathrm{~m}^{3}$, em julho, e $5.167 .124 \mathrm{~m}^{3}$ em dezembro. Ao se adotar eficiência de irrigação de 0,85 , concluiu-se que a lâmina bruta aplicada em todo o PISG seria de $29.532 .142 \mathrm{~m}^{3}$, ou seja, representaria $64 \%$ da capacidade do açude de São Gonçalo. Cabe ressaltar que, no semestre de janeiro a junho, foram realizadas irrigações, mesmo que com menor frequência e volume por planta, correspondentes à metade da aplicada no semestre de julho a dezembro. Esses resultados são indicativos de que os volumes de água destinados à irrigação foram bastante expressivos e que há necessidade de implantação de sistemas de avaliação que assegurem a utilização da água de modo efetivamente sustentável.

\section{Conclusões}

1. As estimativas da evapotranspiração real (ETr) de áreas irrigadas do perímetro irrigado de São Gonçalo, com uso do algoritmo SEBAL, apresentam boa concordância com os valores da evapotranspiração obtidos com o método da FAO.

2. O mapeamento da ETr possibilita identificar padrões que diferenciam áreas irrigadas das não irrigadas, bem como diferenças existentes na ETr no interior do perímetro irrigado de São Gonçalo.

3. O volume de água aplicado nas irrigações do perímetro irrigado de São Gonçalo, no semestre de maior demanda, é superior a $64 \%$ da capacidade de armazenamento de água do reservatório.

\section{Agradecimentos}

Ao Conselho Nacional de Desenvolvimento Científico e Tecnológico, pelo apoio financeiro e pela concessão de bolsas; e ao Instituto Nacional de Meteorologia, pela disponibilização de dados da estação meteorológica de São Gonçalo, PB.

\section{Referências}

ALlEN, R.G.; PEREIRA, L.S.; RAES, D.; SMITH, M. Crop evapotranspiration: guidelines for computing crop water requirements. Rome: FAO, 1998. 300p. (FAO. Irrigation and drainage paper, 56).

ALLEN, R.G.; TASUMI, M.; TREZZA, R. Satellite-based energy balance for mapping evapotranspiration with internalized calibration (METRIC) - model. Journal of Irrigation and Drainage Engineering, v.133, p.380-394, 2007.

AZEVEDO, P.V. de; SOUZA, C.B. de; SILVA, B.B. da; RODRIGUES, V.P.S. Water requirements of pineapple crop grown in a tropical environment, Brazil. Agricultural Water Management, v.88, p.201-208, 2007.

BASTIAANSSEN, W.G.M. SEBAL - based sensible and latent heat fluxes in the irrigated Gediz Basin, Turkey. Journal of Hydrology, v.229, p.87-100, 2000. 
BASTIAANSSEN, W.G.M.; MENENTI, M.; FEDDES, R.A.; HOLSLAG, A.A.M. A remote sensing surface energy balance algorithm for land (SEBAL) - 1. Formulation. Journal of Hydrology, v.212, p.198-212, 1998.

BEZERRA, B.G.; SILVA, B.B. da; FERREIRA, N.J. Estimativa da evapotranspiração real diária utilizando-se imagens digitais TM Landsat 5. Revista Brasileira de Meteorologia, v.23, p.305-317, 2008.

BORGES, C.J.R.; AZEVEDO, P.V. de; SILVA, V. de P.R. da; CAMPOS, J.H.B. da C; MOURA, M.S.B. de; SOARES, J.M.; SILVA, B.B. da. Influência do calor armazenado no sistema solo-planta no balanço de energia em pomar de mangueiras. Revista Brasileira de Engenharia Agrícola e Ambiental, v.12, p.393-399, 2008.

BOS, M.G.; BURTON, M.A.; MOLDEN, D.J. Irrigation and drainage performance assessment: practical guidelines. Cambridge: CABI Publishing, 2005. 166p.

FERERES, E.; SORIANO, M.A. Deficit irrigation for reducing agricultural water use. Journal of Experimental Botany, v.58, p.147-159, 2007.

FOLHES, M.T.; RENNÓ, C.D.; SOARES, J.V. Remote sensing for irrigation water management in the semi-arid Northeast of Brazil. Agricultural Water Management, v.96, p.1398-1408, 2009.

HUFFAKER, R.; WHITTLESEY, N.; MICHELSEN, A.; TAYLOR, R.; MCGUCKIN, T. Evaluating the effectiveness of conservation water-pricing programs. Journal of Agricultural and Resource Economics, v.23, p.12-19, 1998.

KUSTAS, W.P.; NORMAN, J.M. Use of remote sensing for evapotranspiration monitoring over land surfaces. Hydrological Sciences Journal, v.41, p.495-516, 1996.

MARENGO, J.A.; JONES, R.; ALVES, L.M.; VALVERDEA, M.C. Future change of temperature and precipitation extremes in South America as derived from the PRECIS regional climate modeling system. International Journal of Climatology, v.29, p.2241-2255, 2009.

MIRANDA, F.R. de; GOMES, A.R.M.; OLIVEIRA, C.H.C. de; MONTENEGRO, A.A.T.; BEZERRA, F.M. Evapotranspiração e coeficiente de cultivo do coqueiro anão-verde na região litorânea do Ceará. Revista Ciência Agronômica, v.38, p.129-135, 2007.

SANTOS, T.V. dos; FONTANA, D.C.; ALVES, R.C.M. Avaliação de fluxos de calor e evapotranspiração pelo modelo SEBAL com uso de dados do sensor ASTER. Pesquisa Agropecuária Brasileira, v.45, p.488-496, 2010.

SILVA, B.B. da; BEZERRA, M.V.C. Determinação dos fluxos de calor sensível e latente na superfície utilizando imagens TM - Landsat 5. Revista Brasileira de Agrometeorologia, v.14, p.174-186, 2006.

SILVA, B.B. da; LOPES, G.M.; AZEVEDO, P.V. de. Balanço de radiação em áreas irrigadas utilizando imagens Landsat 5 - TM. Revista Brasileira de Meteorologia, v.20, p.243-252, 2005.

SILVA, V. de P.R. da; AZEVEDO, P.V. de; SILVA, B.B. da. Surface energy fluxes and evapotranspiration of a mango orchard grown in a semiarid environment. Agronomy Journal, v.99, p.1391-1396, 2007.

SOBRINO, J.A.; GÓMEZ, M.; JIMÉNEZ-MOÑOZ, J.C.; OLIOSO, A.; CHEHBOUNI, G. A simple algorithm to estimate evapotranspiration from DAIS data: application to the DAISEX campaigns. Journal of Hydrology, v.315, p.117-125, 2005.

SOUSA, I.F.; NETTO, A.O.A.; CAMPECHE, L.F.M.S.; BARROS, A.C.; SILVA, V. de P.R. da; AZEVEDO, P.V. de. Lisímetro de pesagem de grande porte. Parte II: Consumo hídrico do coqueiro anão verde irrigado. Revista Brasileira de Engenharia Agrícola e Ambiental, v.15, p.526-532, 2011.

TIMMERMANS, W.J.; KUSTAS, W.P.; ANDERSON, M.C.; FRENCH, A.N. An intercomparison of the Surface Energy Balance Algorithm for Land (SEBAL) and the Two-Source Energy Balance (TSEB) modeling schemes. Remote Sensing of Environment, v.108, p.369-384, 2007.

VAREJÃO-SILVA, M.A.; BRAGA, C.C.; SILVA, B.B. da. Atlas climatológico do Estado da Paraíba. Campina Grande: UFPB, 1984. Atlas.

ZWART, S.J.; BASTIAANSSEN, W.G.M. SEBAL for detecting spatial variation of water productivity and scope for improvement in eight irrigated wheat systems. Agricultural Water Management, v.89, p.287-296, 2007. 